\title{
Efficient Radar Emitters Scheme Recognition based on a Novel SVM Algorithm
}

\author{
QIANG LIU \\ School of Academic Affairs, Harbin University of Science and Technology, Harbin, P.R. China \\ Liuqiang@hrbust.edu.cn
}

Keywords: one-class svm; Bayesian classifier; radar emitter mod scheme; recognition probabilitiy

\begin{abstract}
This paper proposed a efficient radar emitter signal scheme recognition method using a novel one-class SVM based Bayesian classification algorithm. First, it is proven that the solution of one-class SVM using the Gaussian kernel can be normalized as an estimate of probability density, and the probability density is used to construct the two-class and multi-class Bayesian clusters. The statistical characterization parameters of the multi radar emitter signals are extracted as the input feature vectors of the one-class SVM. Simulation result showed that the correct emitter scheme classification probability of the proposed classifier is comparable to traditional multi-class SVM classifier and better than the ANN based method. However, in the condition of large emitter mod class amount and large amount of training samples of each emitter signal class, the calculation amount of training and storage is only 0.5 percent of the traditional SVM classifier, which lead to less training time for the new classifier. In a word, the new mod scheme recognition method can be widely used in signal recognition field.
\end{abstract}

\section{Introduction}

Radar emitter recognition is an important part of Radar systems, to get the type of radar emitter after selection and feature extraction. Due to the proliferation of environmental electromagnetic sources and noise, radar receivers receive signals amid pollution and interference. And traditional recognition methods are inefficient against the distortion of signal. Radar emitter scheme recognition is increasing in importance and extensive work has been carried out for a number of years in signal processing field.[1] The classic recognition method is pattern recognition and statistics judgment [2][3][4]. Recent published modulation recognition algorithms include artificial neural networks (ANN) and support vector machine (SVM) raised new methods of the digital modulation recognition [5][6]. Generally speaking, the recognition probability of traditional SVM algorithm is higher than ANN algorithm [7]. Although the performance of the traditional SVM based recognition algorithm is good, but it's calculation amount of the training and storage is super large in the condition of multi-class signal. So the traditional SVM recognition method may not fit for online classifying system. To solve the problem, a novel one-class SVM based Bayesian multi-classifier is proposed in this paper. The aim of this work is to develop a novel SVM based algorithm which has the same high recognition probability as traditional SVM classifier and needs more less calculation amount to fit the online software radio recognition task.

Signal modulation scheme recognition method includes feature extraction part and modulation scheme recognition part. Simulation experiments will classify 6 types of radar emitter signals. Here, radar emitter signal feature parameters are extracted from the amplitude, phase, frequency and power spectrum of the signal as traditional methods [2][3][4]. Now the feature extraction process will be presented below.

\section{Feature Extraction}

In signal processing system, a radar emitter signal can be presented as the following expression:

$$
s(t)=A_{m}(t) P\left(2 \pi f_{m}(t)+\Phi_{m}(t)\right)
$$


In equation $(1), \mathrm{A}_{\mathrm{m}}(\mathrm{t})$ denotes the amplitude function of the emitter signal, $\mathrm{P}(\mathrm{t})$ is the radar emitter waveform function, $\mathrm{f}_{\mathrm{m}}(\mathrm{t})$ and $\Phi_{m}(t)$ denote the frequency and phase function of the signal. According to equation (1), some useful feature parameters can be extracted below.

1. Maximum value of the spectral density of zero center normalization momentary amplitude $\gamma_{\max }$ :

$$
\gamma_{\max }=\max \mid D F T\left(\left.a_{c n}(i)\right|^{2} / N_{s}\right.
$$

Here, $\mathrm{N}_{\mathrm{s}}$ denotes the number of the samples, $a_{\mathrm{cn}}(i)=a_{\mathrm{n}}(i)-1, a_{\mathrm{n}}(i)=a(i) / m_{a}, \mathrm{~m}_{\mathrm{a}}$ denotes sample average.

2. Standard deviation of the absolute value of the zero center non-weak section momentary phase nonlinear component $\sigma_{\mathrm{ap}}$ :

$$
\sigma_{a p}=\sqrt{\frac{1}{C}\left(\sum_{a_{n}(i)>a_{t}} \Phi_{N L}^{2}(i)\right)-\left(\frac{1}{C} \sum_{a_{n}(i) a_{t}} \mid \Phi_{N L}(i)\right)^{2}}
$$

Here, $a_{t}$ denotes amplitude circumscription level to judge non-weak section momentary of the signal, $C$ denotes the number of the non-weak section momentary phase nonlinear component in all samples $\mathrm{N}_{\mathrm{s}} . \Phi_{N L}(i)$ denotes the zero center normalization momentary phase nonlinear component. In carrier synchronization conditions we can get such equation:

$\Phi_{N L}(i)=\Phi(i)-\Phi_{0}=\Phi_{N L}(i)-\frac{1}{N_{s}} \sum_{i=1}^{N_{s}} \Phi(i), \Phi(i)$ is momentary phase.

3. Standard deviation of the zero center non-weak section momentary phase nonlinear component $\sigma_{\mathrm{dp}}$ :

$$
\sigma_{d p}=\sqrt{\frac{1}{C}\left(\sum_{a_{n}(i)>a_{t}} \Phi_{N L}^{2}(i)\right)-\left(\frac{1}{C} \sum_{a_{n}(i)>a_{t}} \Phi_{N L}(i)\right)^{2}}
$$

4. Standard deviation of the absolute value of the zero center normalization momentary amplitude $\sigma_{\mathrm{aa}}$ :

$$
\sigma_{a a}=\sqrt{\frac{1}{N_{s}}\left(\sum_{i=1}^{N_{s}} a_{c n}^{2}(i)\right)-\left(\frac{1}{N_{s}} \sum_{i=1}^{N_{s}} \mid a_{c n}(i)\right)^{2}}
$$

5. Standard deviation of the absolute value of the zero center normalization non-weak section momentary frequency nonlinear component $\sigma_{\mathrm{af}}$ :

$$
\sigma_{a f}=\sqrt{\frac{1}{C}\left(\sum_{a_{n}(i)>a_{t}} f_{N}^{2}(i)\right)-\left(\frac{1}{C} \sum_{a_{n}(i)>a_{t}} f_{N}(i)\right)^{2}}
$$

Where:

$f_{N}(i)=\frac{f_{m}(i)}{R_{s}}=\frac{f(i)-m_{f}}{R_{s}}=\frac{f(i)-\frac{1}{N_{s}} \sum_{i=1}^{N_{s}} f(i)}{R_{s}}, \mathrm{R}_{\mathrm{s}}$ denotes character rate of the modulation signal, $f(i)$ denotes momentary frequency.

\section{One-class SVM based Multi-class Bayesian Algorithm}

The novel bayesian algorithm can be distributed as two steps. First, the gaussian kernel one-class SVM will be proven to be an estimate of probability density. Second, the probability density can be used to construct the two-class and multi-class Bayesian classifier.

\section{One-class SVM based Probability DensityEstimation}

One-class SVM was firstly proposed by Scholkopf to estimate the minimal support field [8]. Suppose the nonlinear mapping function $\Phi(x)$ as the feature mapping of kernel function $K(x, x)$. One-class SVM constructs the hyperplane in higher dimentional feature space to separate function set $\left\{\Phi\left(x_{1}\right), \Phi\left(x_{2}\right), \ldots, \Phi\left(x_{l}\right)\right\}$ and origin and make the hyperplane mostly further to origin[9][10]. It can be described as a quadratic programming problem in math $(0 \leq v \leq 1)$ :

$$
\begin{gathered}
\min \left\{\frac{1}{2}\|w\|^{2}+\frac{1}{v l} \sum_{i=1}^{l} \xi-\rho\right\} \\
\text { s.t. }\left\langle w, \Phi\left(x_{i}\right)\right\rangle \geq \rho-\xi_{i},\left(\xi_{i} \geq 0, \rho \geq 0\right)
\end{gathered}
$$

The solution of (7) is:

$$
f_{1}(x)=\sum_{i} \alpha_{i} K\left(x_{i}, x\right)-\rho^{*}, \alpha_{i} \geq 0 .
$$


If $\alpha_{i}>0, x_{i}$ is support vector. If the goal is to estimate the support field only contains training samples, we can consider that all solutions which make $f_{l}(x)>0$ is in this field .

Here we use Gaussian kernel function:

$$
\mathrm{K}\left(\mathrm{x}, \mathrm{x}^{\prime}\right)=\exp \left(-\left\|\mathrm{x}-x^{\prime}\right\|^{2} / 2 \sigma^{2}\right), \beta>0
$$

Erase $-\rho^{*}$ from $f_{1}(x)$, we define function $f(x)$ as:

$$
f(x)=\sum_{i} \alpha_{i} K\left(x_{i}, x\right), \alpha_{i} \geq 0 .
$$
$f(x)$ :

Function $f(x)$ can be proved to be normalized as probability density function. Normalizing function

$$
f^{*}(x)=\frac{\sum_{i} \alpha_{i} K\left(x_{i}, x\right)}{\int \sum_{i} \alpha_{i} K\left(x_{i}, x\right) d x},
$$

Here we know $f^{*}(x) \geq 0$ and

$$
\int f^{*}(x) d x=1
$$

So $f^{*}(x)$ is a probability density estimation function, and it can be used to construct optimal Bayesian classifier.

\section{One-class SVM based Bayesian classifier}

Now the probability density estimated by one-class SVM will be used to construct Bayesian classifier. Here, we assume the class number of the samples is $C$. To each class, the samples is trained by one-class SVM, the solutions (classification function, $i=1,2, \ldots, C$ ) is:

$$
f^{i}(x)=\sum_{k} \alpha_{k}^{i} K^{i}\left(x_{k}^{i}, x\right), \alpha_{k}^{i} \geq 0 .
$$

Thus we can get:

$$
\mathrm{p}(x \mid i)=\frac{f^{i}(x)}{\int f^{i}(x) d x}
$$

Equation (14) can be considered as the conditional probability estimation of class $i$, and the prior probability is:

$$
\mathrm{P}(i)=\frac{\int f^{i}(x)}{\sum_{j=1}^{C} \int f^{j}(x) d x}
$$

Equation (15) is prior probability estimation of class $i$. According to Bayesian theory, the classification process is the process of comparing $p(x \mid i) P(i)(i=1,2, \ldots, C)$ :

$$
\mathrm{p}(\mathrm{x} \mid i) \mathrm{P}(i)=\frac{f^{i}(x)}{\sum_{j=1}^{c} \int f^{j}(x) d x}
$$

To a feature vector sample $x$ ( $x$ is to be classified, the probable class number is $C$ ) : if $p(x \mid i) P(i)=\max \{p(x \mid j) P(j)\}, j=1, \ldots, C$, then $x \in i$.

After simplifying the denominator, the classification process is the process of comparing $f(x)$, just the solutions of one-class SVM of each class samples. Now the classification principle of one-class SVM based Bayesian algorithm is proposed here:

To a feature vector sample $x$ ( $x$ is to be classified, the probable class number is $C$ ): if $f^{i}(x)=\max \left\{f^{\prime}(x), j=1,2, \ldots, C\right\}, j=1, \ldots, C$, then $x \in i$. 


\section{Advantages of the One-class SVM based Bayesian Multi-classification Algorithm}

Compare with traditional SVM multi-classifier, the advantage of the proposed algorithm is that the proposed algorithm needs less calculation amount and less storage space. Assume that the sample's amount of each training set is $P$. Each traditional SVM needs to calculate any two kernels' inner product in each class kernel mixes. Thus the calculation amount is:

$$
C P(C P+1) / 2
$$

And the calculation amount of $C$ class classifier is:

$$
C[C P(C P+1)] / 2
$$

To one-class SVM based Bayesian classifier, it only needs to calculate any two kernels' inner product in only one class kernel mix. Thus the $C$ class's classification calculation amount is:

$$
C[P(P+1)] / 2
$$

According to the above analysis, kernel inner product calculation amount (same as the storage space) of the traditional SVM multi-classifier is $\left(C^{3} P^{2}+C^{2} P-C P^{2}-C P\right) / 2$ more than that of one-class SVM based Bayesian classifier.

The calculation amounts in different class number $C$ and sample amount $p$ of each class of the traditional SVM classifier and one-class SVM based Bayesian classifier are shown in TABLE I and TABLE II . And the two algorithms' calculation amount difference is shown in TABLEIII. Figure 1 shows the comparison of training calculation amounts between the two algorithms (Traditional SVM classifier is abbreviated as TSVM algorithm and one-class SVM based Bayesian classifier is abbreviated as OCSB algorithm in the following text and figure 1).

Table 1 Kernel calculation amounts of multi traditional two-class SVM based multi classifier

\begin{tabular}{|l|l|l|l|}
\hline & \multicolumn{1}{|c|}{$\mathbf{P}=\mathbf{1 0 0}$} & \multicolumn{1}{|c|}{$\mathbf{P}=\mathbf{3 0 0}$} & \multicolumn{1}{|c|}{$\mathbf{P}=\mathbf{6 0 0}$} \\
\hline $\mathrm{C}=3$ & 135450 & 1216350 & 4862700 \\
\hline $\mathrm{C}=6$ & 1081800 & 9725400 & 38890800 \\
\hline $\mathrm{C}=10$ & 5005000 & 45015000 & 180030000 \\
\hline $\mathrm{C}=15$ & 16886250 & 151908750 & 607567500 \\
\hline
\end{tabular}

Table 2 Kernel calculation amounts of one-class SVM based Bayesian multi classifier

\begin{tabular}{|l|c|c|c|}
\hline & $\mathbf{P}=\mathbf{1 0 0}$ & $\mathbf{P}=\mathbf{3 0 0}$ & \multicolumn{1}{|c|}{$\mathbf{P}=\mathbf{6 0 0}$} \\
\hline $\mathrm{C}=3$ & 15150 & 135450 & 540900 \\
\hline $\mathrm{C}=6$ & 30300 & 270900 & 1081800 \\
\hline $\mathrm{C}=10$ & 50500 & 451500 & 1803000 \\
\hline $\mathrm{C}=15$ & 75750 & 677250 & 2704500 \\
\hline
\end{tabular}

Table 3 Kernel calculation differences of two algorithms

\begin{tabular}{llll} 
& \multicolumn{1}{c}{$\mathbf{P}=\mathbf{1 0 0}$} & \multicolumn{1}{c}{$\mathbf{P}=\mathbf{3 0 0}$} & \multicolumn{1}{c}{$\mathbf{P}=\mathbf{6 0 0}$} \\
$\mathrm{C}=3$ & 120300 & 1080900 & 4321800 \\
$\mathrm{C}=6$ & 1051500 & 9454500 & 37809000 \\
$\mathrm{C}=10$ & 4954500 & 44563500 & 178227000 \\
$\mathrm{C}=15$ & 16810500 & 151231500 & 604863000
\end{tabular}

Shown from TABLE I to TABLE III and figure 1, in the condition of each class has the same training sample amount $P$, with the increase of the class number $C$, the traditional SVM algorithm's kernel inner product calculation amount increases with the speed of $C^{3}$, and that of one-class SVM based Bayesian algorithm increases with the speed of $C$. Compared with the traditional SVM algorithm the proposed algorithm has huge advantage in calculation amount in the condition of big class number $C$ to be classified and large training samples amount $P$ of each class. In the condition of $C=15$ and $P=600$, the traditional SVM algorithm's calculation amount is more than 600 million, and the one-class SVM based Bayesian algorithm's calculation is only 2.7 million (it is $0.5 \%$ of traditional SVM algorithm's calculation amount). 


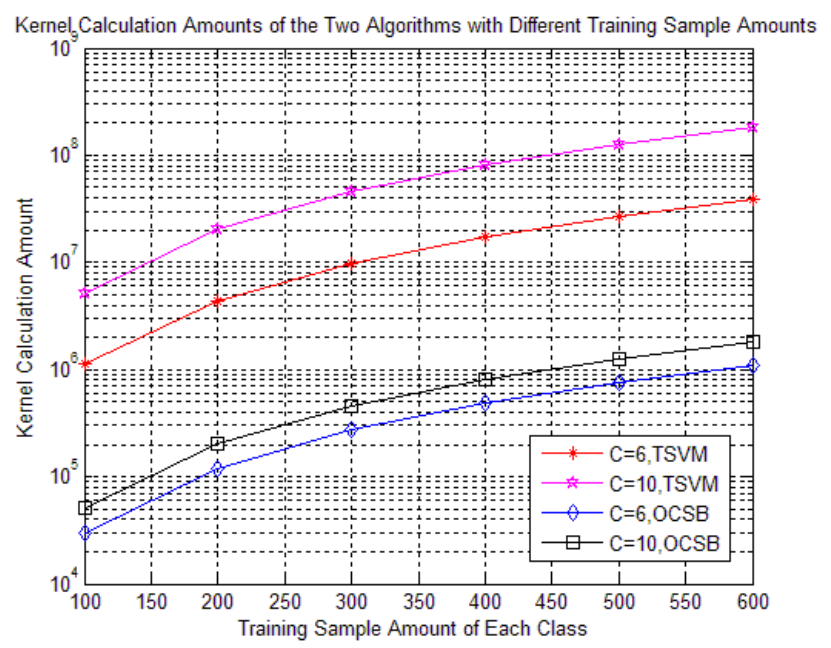

Figure 1. Comparison of training calculation amounts between two algorithms

\section{Algorithm radar emitter scheme recognition experiment}

After extracting the feature vector of the radar emitter signal and contracting the OCSB algorithm. Radar emitter scheme recognition experiment result using OCSB algorithm is proposed in this part. To test whether the recognition performance is satisfying, recognition experiment result of the same sample signal using TSVM algorithm is also proposed as a comparison.

The radar emitter signal is generated by random signal. The center frequency of the receiver is range from 8.7-9.5GHz, and 6 different radar emitter schemes (R1 to R6) using different modulation type are generated in different experiment SNR conditions $(5 \mathrm{~dB}, 10 \mathrm{~dB}, 15 \mathrm{~dB}, 20 \mathrm{~dB})$ to train the one-class SVM and traditional SVM. Number of each emitter type's training data samples in each SNR condition is 100. After the training process and getting the solutions of each one-class SVM, 400 testing signals of each emitter type in each SNR condition is generated to test the recognition probability. And the same training and testing process is also carried out using traditional SVM algorithm. Cross validation tests is carried out to get the average recognition probability of the two algorithms. Experiment result is shown in TABLE IV.

Table 4

COMPARISON OF EMITTER SCHEME RECOGNITION PROBABILITY OF TWO ALGORITHMS

\begin{tabular}{|c|c|c|c|c|c|c|}
\hline \multirow{2}{*}{$\begin{array}{c}\text { SNR \& } \\
\text { Algorithm }\end{array}$} & \multicolumn{6}{|c|}{ Radar Emitter Recognition Probability } \\
\hline & $R \mathbf{R}$ & $R 2$ & $R 3$ & $R 4$ & $R 5$ & $R 6$ \\
\hline $5 \mathrm{~dB}(\mathrm{OC})$ & 98.5 & 98.8 & 96.5 & 97.3 & 97.5 & 97.9 \\
\hline $5 \mathrm{~dB}(\mathrm{TS})$ & 98.9 & 99.2 & 96.8 & 97.2 & 97.3 & 97.7 \\
\hline $10 \mathrm{~dB}(\mathrm{OC})$ & 99.1 & 100 & 97.2 & 97.3 & 97.6 & 97.9 \\
\hline $10 \mathrm{~dB}(\mathrm{TS})$ & 99.3 & 100 & 97.1 & 97.5 & 97.7 & 97.8 \\
\hline $15 \mathrm{~dB}(\mathrm{OC})$ & 100 & 100 & 97.8 & 97.9 & 97.9 & 98.1 \\
\hline $15 \mathrm{~dB}(\mathrm{TS})$ & 100 & 100 & 98.1 & 98.1 & 97.8 & 98.1 \\
\hline $20 \mathrm{~dB}(\mathrm{OC})$ & 100 & 100 & 98.8 & 98.6 & 98.1 & 98.4 \\
\hline $20 \mathrm{~dB}(\mathrm{TS})$ & 100 & 100 & 98.7 & 98.5 & 98.1 & 98.5 \\
\hline
\end{tabular}

Seen from TABLE IV, the recognition probability of OCSB(OC) algorithm is proximal to TSVM(TS) algorithm in all SNR conditions. So the OCSB and the TSVM algorithm both show good recognition performance in low SNR condition. Thus the calculation amount excellence brings the OCSB algorithm super advantage in on-line modulation scheme recognition condition in radar emitter clustering application. The number of the radar emitter signal class is $C=6$ and the amount of each training sample of each signal class is $P=100$. From TABLE I to TABLE III, we know the calculation amount of the OCSB algorithm is 30300 , and the calculation amount of the TSVM algorithm is 1081800. The OCSB algorithm has huge advantage in calculation, so it may be widely used in multi-class radar emitter recognition. 


\section{Conclusions}

A novel one-class SVM based Bayesian algorithm is proposed to recognize the emitter scheme of multi-class communication signals. The statistical characterization parameters of the radar emitter signals are extracted as the training feature vector samples of one-class SVM. The solution of the one-class SVM is used to construct the Bayesian classifier. Simulation experiments show that the recognition probability of the proposed OCSB algorithm is no less than that of TSVM algorithm. However, comparing with the TSVM algorithm, the OCSB algorithm has huge advantage in kernel inner product calculation amount (in the case of class number $C=15$ and each class's training amount $P=600$, the calculation amount of the proposed OCSB algorithm is only $0.5 \%$ of the calculation amount of the TSVM algorithm), which leads to less training time and less storage space for the proposed classifier, and can be widely used in on-line recognition radar emitter systems.

\section{References}

[1] Bezousek P., and Schejbal V., 2004, "Radar Technology in the Czech Republic," IEEE Aerospace and Electronic Systems Magazine, Vol. 19, No.8, pp. 27-34,2004.

[2] Polydoros A and Kim K, "On the Detection and Classification of Digital Modulations in Broad-band Noise ," IEEE Transaction on Communication, 38(8): -1199-1211, 1990.

[3] Dominguez L and Borrallo J, "A General Approach to the Automatic Classification of Radio Communication Signals," Signal Processing, 22(3):239-250, 1991.

[4] Nandi A K and Azzouz E E, "Automatic Modulation Recognition," Signal Processing, 46(2):211-222, 1995.

[5] Yin Zhendong, Yang Wei, Yang Zhutian, Zuo Lihua and Gao hansong. "A Study on Radar Emitter Recognition based on SPDS Neural Network". Information Technology Journal, 2011, (4) :883-888.

[6] Platt J, Crisianini N and Taylor J, "Large Margin DAG's for Multiclass Classification," Advances in Neural Information Processing Systems, Cambridge, USA: MIT Press : 547-553, 2000.

[7] Jiancheng Sun, Taiyi Zhang and Feng Liu, "Automatic Multi-class Digital Modulation Recognition Algorithm based on Support Vector Machine," Journal of Xian Jiaotong University, 38(6):619-622, 2004

[8] Scholkopf B, Platt J and Shawe-Taylor J, "Estimating the support of a high-dimensional distribution," Neural Computation, 13(7):1443-1471, 2001.

[9] Camastra F and Verri A, "A Novel Kernel Method for Clustering," IEEE Transactions on Parttern Recognition Analysis and Machine Intelligence, 27(5): 801-805, 2005.

[10]Cristianini N and Shawe-Taylor J. "An Introduction to Support Vector Machines and Other Kernel based Learning Methods," Cambridge University Press, 2000. 